PREPARED FOR SUBMission to JHEP

\title{
Constraining BSM Physics at the LHC: Four top final states with NLO accuracy in perturbative QCD
}

\author{
G. Bevilacqua ${ }^{a}$ and M. Worek ${ }^{b}$ \\ ${ }^{a}$ Institut für Theoretische Teilchenphysik und Kosmologie, RWTH Aachen University, Otto-Blumenthal \\ Str., D-52056 Aachen, Germany \\ ${ }^{b}$ Theoretische Physik, Fachbereich C, Bergische Universität Wuppertal, Gauss Str. 20, D-42097 \\ Wuppertal, Germany \\ E-mail: bevilacqua@physik.rwth-aachen.de, \\ worek@physik. uni-wuppertal.de
}

Abstract: Many theories, from Supersymmetry to models of Strong Electroweak Symmetry Breaking, look at the production of four top quarks as an interesting channel to evidentiate signals of new physics beyond the Standard Model. The production of four-top final states requires large partonic energies, above the $4 m_{t}$ threshold, that are available at the CERN Large Hadron Collider and will become more and more accessible with increasing energy and luminosity of the proton beams. A good theoretical control on the Standard Model background is a fundamental prerequisite for a correct interpretation of the possible signals of new physics that may arise in this channel. In this paper we report on the calculation of the next-to-leading order QCD corrections to the Standard Model process $p p \rightarrow t \bar{t} t \bar{t}+X$. As it is customary for such studies, we present results for both integrated and differential cross sections. A judicious choice of a dynamical scale allows us to obtain nearly constant $\mathcal{K}$-factors in most distributions.

Keywords: NLO Computations, Heavy Quark Physics, Standard Model, Beyond Standard Model 


\section{Contents}

1 Introduction 1

2 Details of the calculation $\quad 2$

3 Predictions for the LHC $\quad \mathbf{5}$

3.1 Integrated cross section and its scale dependence for $\mu_{0}=2 m_{t} \quad 5$

$\begin{array}{lll}3.2 & \text { Differential cross sections for } \mu_{0}=2 m_{t} & 7\end{array}$

3.3 Integrated cross section and its scale dependence for $\mu_{0}=H_{T} / 4 \quad 9$

$\begin{array}{lr}3.4 \text { Differential cross sections for } \mu_{0}=H_{T} / 4 & 10\end{array}$

4 Summary and Conclusions $\quad 16$

\section{Introduction}

Since its discovery in 1995 by the CDF and D0 experiments at Fermilab, the top quark, the most massive of all the observed elementary particles, has been extensively scrutinized. Several properties and observables have been already analysed including, among others, the top-pair and single-top production cross sections together with the measurement of the top quark mass. Most of these measurements have been, however, limited by the relatively small statistics collected at the Tevatron. With a much higher center of mass energy and luminosity, the Large Hadron Collider (LHC) has all the features of a top factory and will significantly add to the previous measurements in this field. At the nominal design value of the beam energy $(\sqrt{s}=14 \mathrm{TeV})$ and assuming a luminosity of $10 \mathrm{fb}^{-1} /$ year, the LHC will collect about 8 million top anti-top pair events and another few million tops via electroweak single top quark production. In fact even at present, running at $\sqrt{s}=7$ $\mathrm{TeV}$, it has made possible to start re-examining the main properties of the top quark with significant precision.

The large statistics available will open a window on entirely new measurements as well as on the analysis of novel, more complex final states. As to $t \bar{t} t \bar{t}$ production, the LHC energy is sufficient to produce such events at a sensible rate. Assuming only the standard, dominant $t \rightarrow W b$ decay, this process leads to $W^{+} W^{-} W^{+} W^{-} b \bar{b} b \bar{b}$ final states with leptonic and/or hadronic decays of gauge bosons. The experimental signature of such final states is characterized by the presence of opposite and same-sign charged leptons, large missing energy and jets with a considerable number of b-jets. These signatures are precisely the ones that are expected in the Higgs boson(s) and new physics searches. Thus, $t \bar{t} t \bar{t}$ production is an interesting channel to probe several realizations of Standard Model extensions at the LHC. Some prominent examples include models of Higgs and top compositeness as well as models involving the production of new colored resonances with large couplings to the 
top quark. In the latter case, the most studied ones are Kaluza-Klein gluons from the Randall-Sudrum warped extra dimensions. Many of these models predict effective four-top quark interactions as well as new processes such as $p p \rightarrow G G$ and $p p \rightarrow t \bar{t} G$, where $G$ is a new heavy particle decaying to $t \bar{t}$, leading to $t \bar{t} t \bar{t}$ final states (see e.g. [1-10] and references therein). In this context, a precise theoretical description of the four-top production rate in the Standard Model may help to constrain new physics scenarios.

In addition, $t \bar{t} t \bar{t}$ is a major background for many processes arising from supersymmetric extensions of the Standard Model, the most noticeable example being the production of a heavy Higgs boson $[11,12]$. An accurate theoretical description of four-top production would help in this case to determine the Higgs boson self coupling and thus contribute to better understand the Higgs boson potential in this minimal SM extension. Furthermore, multi-top final states can also be produced via long cascade decays of colored superymmetric particles, such as squarks or gluinos [13, 14].

Last but not least, $t \bar{t} t \bar{t}$ production is the last process in the so-called Les Houches nextto-leading order (NLO) experimenter's wishlist [15] that is still waiting for a calculation. Recent breakthrough in one-loop calculational techniques, sometimes referred to as "the unitary revolution" [16-19], together with great improvement in more traditional methods, have led to a tremendous progress in the calculation of multi-leg processes at hadron colliders. This is exemplified by the calculation of the following $2 \rightarrow 4$ processes: $p p(p \bar{p}) \rightarrow$ $t \bar{t} b \bar{b}+X[20-23], p p(p \bar{p}) \rightarrow t \bar{t} j j+X[24,25], p p(p \bar{p}) \rightarrow W^{+} W^{-} b \bar{b}+X[26,27], p p \rightarrow b \bar{b} b \bar{b}+X$ [28], $p p(p \bar{p}) \rightarrow W^{+} W^{-} j j+X[29,30], p p \rightarrow W^{+} W^{+} j j+X[31,32], p p(p \bar{p}) \rightarrow W+3 j+X$ $[33,34], p \bar{p} \rightarrow Z / \gamma^{*}+3 j+X[35], p p \rightarrow W \gamma \gamma j+X[36]$ and $p p \rightarrow 4 j+X$ [37]. In addition, the first NLO QCD corrections to $2 \rightarrow 5$ processes, i.e. $p p \rightarrow W+4 j+X$ and $p p \rightarrow Z / \gamma^{*}+4 j+X$ have recently been completed [38, 39].

In this paper we present the results of a calculation of $p p \rightarrow t \bar{t} t \bar{t}+X$ in the Standard Model at NLO QCD accuracy. This calculation makes the Les Houches wishlist finally complete, with at least one independent calculation available for each benchmark process. The paper is organized as follows. In Section 2 we briefly describe the details of our calculation. Numerical results for the integrated and differential cross sections are presented in Section 3. Finally, in Section 4 we give our conclusions.

\section{Details of the calculation}

At the leading order (LO) in perturbative expansion, $t \bar{t} t \bar{t}$ final states are produced via the scattering of either two gluons or one quark and the corresponding anti-quark. A representative set of Feynman diagrams contributing to the $p p \rightarrow t \bar{t} t \bar{t}$ process at $\mathcal{O}\left(\alpha_{s}^{4}\right)$ is depicted in Figure 1. In total, there are $72 \mathrm{LO}$ diagrams for $g g \rightarrow t \bar{t} t \bar{t}$ and 14 for $q \bar{q} \rightarrow t \bar{t} t \bar{t}$. Even though we do not actually employ Feynman diagrams, it is customary to present them as a measure of the complexity of the calculation. The calculation of scattering amplitudes is based on well-known off-shell iterative algorithms [40-42], performed automatically within the Helac-Dipoles package [43] and cross checked with the Helac-Phegas Monte Carlo program [44-46]. A perfect agreement has been found in all cases. Phase-space optimization and integration has been performed with the help of PARNI [47] and KALEU [48]. 

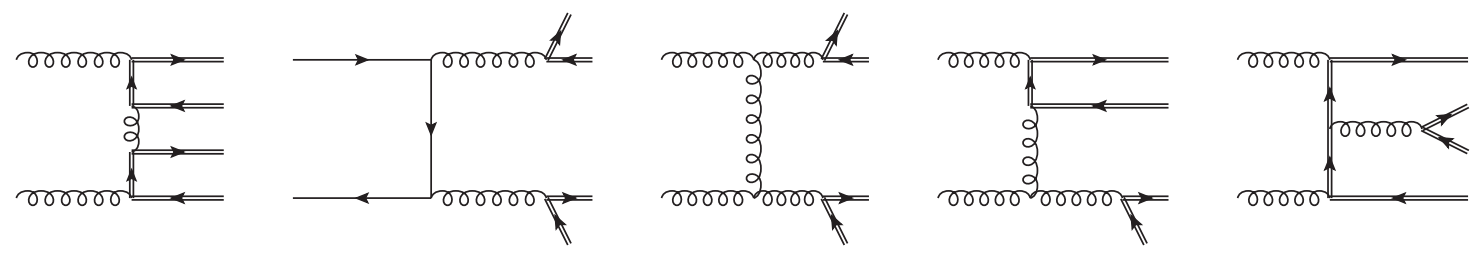

Figure 1. A representative set of Feynman diagrams contributing to the $p p \rightarrow t \bar{t} t \bar{t}$ process at $\mathcal{O}\left(\alpha_{s}^{4}\right)$. Double lines correspond to top quarks, single lines to light quarks and wiggly ones to gluons.
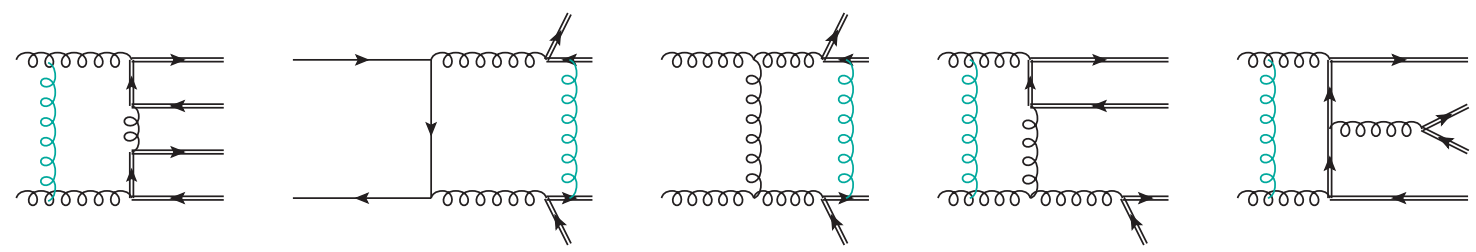

Figure 2. A representative set of pentagon and hexagon diagrams for the $p p \rightarrow t \bar{t} t \bar{t}+X$ process at NLO QCD. Double lines correspond to top quarks, single lines to light quarks and wiggly ones to gluons.
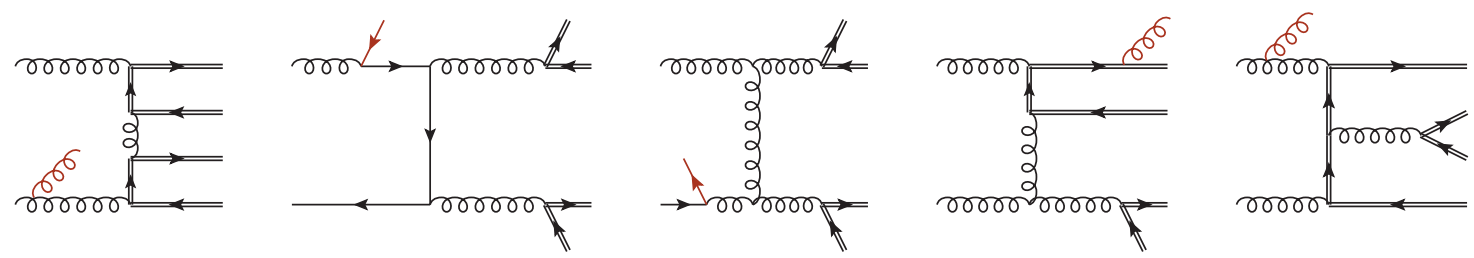

Figure 3. A representative set of Feynman diagrams contributing to the real emission corrections to the $p p \rightarrow t \bar{t} t \bar{t}+X$ process at $\mathcal{O}\left(\alpha_{s}^{5}\right)$. Double lines correspond to top quarks, single lines to light quarks and wiggly ones to gluons.

At the NLO level, the virtual corrections are obtained from the interference of the oneloop diagrams with the tree level amplitude. They can be classified into self-energy, vertex, box-type, pentagon-type and hexagon-type corrections. A representative set of pentagon and hexagon diagrams is shown in Figure 2. The number of one-loop Feynman diagrams for the $p p \rightarrow t \bar{t} t \bar{t}$ process, as obtained with QGRAPH [49], is 2200 for $g g \rightarrow t \bar{t} t \bar{t}$ and 410 for the $q \bar{q} \rightarrow t \bar{t} t \bar{t}$. Virtual corrections are evaluated in $d=4-2 \epsilon$ dimensions in the 't HooftVeltman version of the dimensional regularization scheme. The singularities coming from infrared divergent pieces are canceled by the corresponding ones arising from the so-called integrated dipoles, i.e. from the counterterms of the adopted subtraction scheme integrated over the phase space of the unresolved parton. The finite contributions of the loop diagrams are evaluated numerically in $d=4$ dimension. Finally, to ensure numerical stability, we check Ward identities for every phase space point. The calculation of the virtual corrections 


\begin{tabular}{|c|c|c|}
\hline $\begin{array}{l}\text { PARTONIC } \\
\text { SubProcess }\end{array}$ & $\begin{array}{c}\text { Number Of FEYNMAN } \\
\text { DiagRAMS }\end{array}$ & $\begin{array}{l}\text { NuMBER OF } \\
\text { Dipoles }\end{array}$ \\
\hline$g g \rightarrow t \bar{t} t \bar{t} g$ & 682 & 30 \\
\hline$q \bar{q} \rightarrow t \bar{t} t \bar{t} g$ & 128 & 30 \\
\hline$g q \rightarrow t \bar{t} t \bar{t} q$ & 128 & 10 \\
\hline$q g \rightarrow t \bar{t} t \bar{t} q$ & 128 & 10 \\
\hline
\end{tabular}

Table 1. Partonic subprocesses contributing to the subtracted real emissions at $\mathcal{O}\left(\alpha_{s}^{5}\right)$ for the $p p \rightarrow t \bar{t} t \bar{t}+X$ process. Also, the number of Feynman diagrams and the number of Catani-Seymour dipoles corresponding to these subprocesses are presented.

is achieved with the help of the package HELAC-1Loop [50] which incorporates CuTTools $[51,52]$ and OneLOop [55] as cornerstones. The first code contains an implementation of the OPP method for the reduction of one-loop amplitudes at the integrand level, while the second one is dedicated to the evaluation of the one-loop scalar functions. Renormalization is done, as usual, by evaluating tree-level diagrams with counterterms. For our process, we choose to renormalize the coupling in the $\overline{\mathrm{MS}}$ scheme with five active flavors and the top quark decoupled. The mass renormalization is performed in the on-shell scheme.

The real emission corrections to the LO process arise from tree-level amplitudes with one additional parton, i.e. an additional gluon, or a quark anti-quark pair replacing a gluon. All possible contributions can be classified into the four categories presented in Table 1, together with the number of Feynman diagrams and the Catani-Seymour dipoles corresponding to each subprocess. Typical examples of the real emission graphs are displayed in Figure 3.

For the calculation of the real emission contributions, the package HelaC-DipoleS [43] is employed. It implements the massless dipole formalism of Catani and Seymour [56], as well as its massive version as developed by Catani, Dittmaier, Seymour and Trocsanyi [57], for arbitrary helicity eigenstates of the external partons. A phase space restriction on the contribution of the dipoles as introduced by Nagy and Trocsanyi [58] is also included. For the real corrections as well, we adopt KALEU equipped with additional, special dipoles channels that proved to be important for phase-space optimization in the subtracted real emission part [26].

To summarize, our computational system relies on HeLAC-1Loop and HELAC-DiPOLES that are both parts of the HELAC-NLO framework for NLO QCD calculations [59]. The framework is publicly available ${ }^{1}$. All numerical results are obtained using the same methods as presented in our previous work, therefore we will not describe them here in more detail. Rather, we address the interested reader to our earlier publications in this field [2226]. We would like to emphasize, however, that all parts of the calculation are performed in a completely automatic and fully numerical way.

\footnotetext{
${ }^{1}$ http://helac-phegas.web.cern.ch/helac-phegas/
} 


\begin{tabular}{||c|c|c|c|c|c||}
\hline \hline PROCESS & $\sigma_{\mathrm{LO}}[\mathrm{fb}]$ & $\sigma_{\mathrm{NLO}}^{\alpha_{\max }=1}[\mathrm{fb}]$ & $\sigma_{\mathrm{NLO}}^{\alpha_{\max }=0.01}[\mathrm{fb}]$ & K-FACTOR & {$[\%]$} \\
\hline \hline$p p \rightarrow t \bar{t} t \bar{t}+X$ & $12.056(6)$ & $15.33(2)$ & $15.35(3)$ & 1.27 & 27 \\
\hline \hline
\end{tabular}

Table 2. Integrated cross section at LO and NLO for $p p \rightarrow t \bar{t} t \bar{t}+X$ production at the LHC with $\sqrt{s}=14 \mathrm{TeV}$. Results for the MSTW2008 PDF set are presented. In the last two columns the K factor, defined as the ratio of the NLO cross section to the respective $L O$ result, and NLO corrections in $\%$ are given. The scale choice is $\mu_{F}=\mu_{R}=\mu_{0}=2 m_{t}$.

\section{Predictions for the $\mathrm{LHC}$}

We consider the process $p p \rightarrow t \bar{t} t \bar{t}+X$ at the LHC with a nominal design center of mass energy of $\sqrt{s}=14 \mathrm{TeV}$. For the top quark mass, we use the Tevatron average value $m_{t}$ $=173.2 \mathrm{GeV}$ as measured by the $\mathrm{CDF}$ and D0 experiments. Our calculation, like any fixed-order one, embodies a residual dependence on the renormalization scale $\left(\mu_{R}\right)$ and the factorization scale $\left(\mu_{F}\right)$ arising from truncation of the perturbative expansion. As a consequence, the value of observables depends on the values of $\mu_{R}$ and $\mu_{F}$ that are provided as input parameters. For many processes, a natural scale can be easily identified in several ways. For example, one may consider the mass of the heavy particle appearing in the process, or even the typical momentum transfer or the total transverse energy of the process. In the case of $t \bar{t} t \bar{t}$, setting $\mu_{0}=\mu_{R}=\mu_{F}=2 m_{t}$ seems a quite natural scale choice. In fact, when considering total cross sections, effects of the phase space regions close to the top production threshold are expected to dominate, which justifies our choice.

We consider the MSTW2008 set of parton distribution functions [60] as our default PDF set. In particular, we take MSTW2008lo68cl PDFs with 1-loop running $\alpha_{s}$ at LO and MSTW2008nlo68cl PDFs with 2-loop running $\alpha_{s}$ at NLO, including five active flavors. The strong coupling constant is provided by the PDF set itself. It should be noticed that the contribution induced by bottom-quark densities amounts to $0.05 \%$ at the LO. We decided therefore to neglect the contribution of the $b \bar{b}$ initial state in the computation of the total cross section at both LO and NLO. Outgoing top and anti-top quarks are treated on-shell without any cut restriction on them. At NLO, an additional final-state parton arises. Since this parton has no other partons in the final state to be recombined with (the tops are always assumed to be tagged), it will be the only responsible for the possible additional jet. As a consequence, the properties of the resulting jet are independent of the choice of the jet algorithm and the size of the cone radius. We set no restriction on the kinematics of the extra jet.

\subsection{Integrated cross section and its scale dependence for $\mu_{0}=2 m_{t}$}

We start our presentation of the results with the integrated cross sections. In Table 2 cross sections at LO and NLO for $p p \rightarrow t \bar{t} t \bar{t}+X$ production at the LHC with $\sqrt{s}=14 \mathrm{TeV}$, are presented. Our results for $\mu_{0}=2 m_{t}$ scale using the MSTW2008 PDF set are 

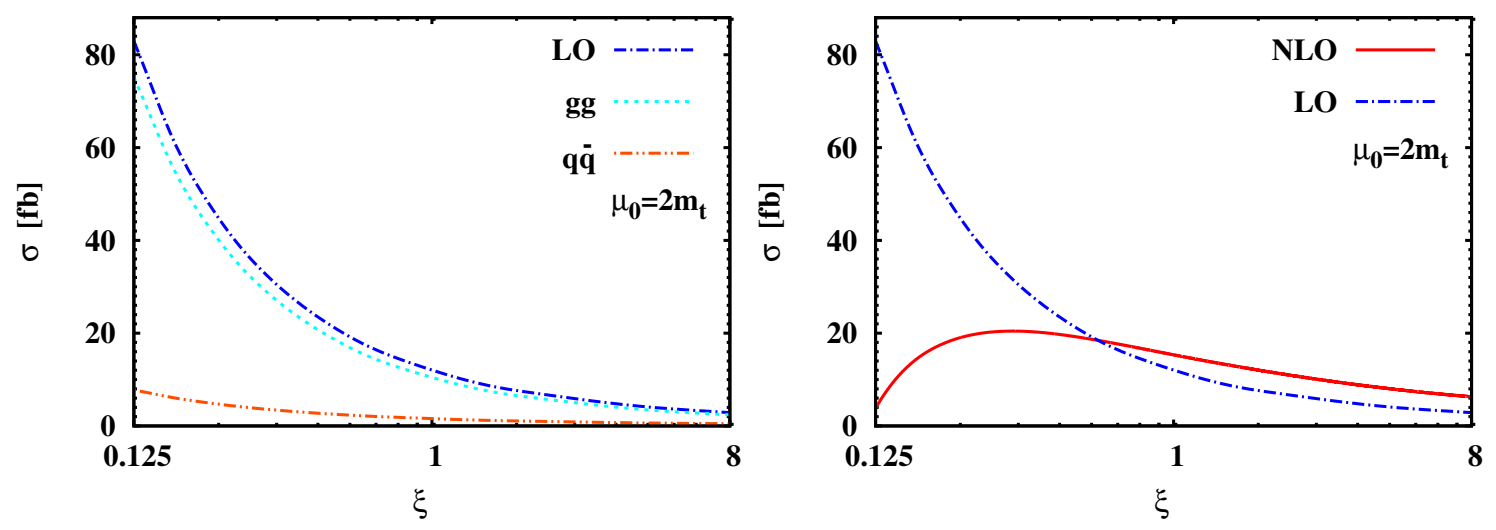

Figure 4. Scale dependence of the LO cross section with the individual contributions of the partonic channels (left panel) and scale dependence of the $L O$ and NLO cross sections (right panel) for the $p p \rightarrow t \bar{t} t \bar{t}+X$ process at the $L H C$ for $\sqrt{s}=14 \mathrm{TeV}$. The scale is set to a common value $\mu_{R}=\mu_{F}=\xi \cdot \mu_{0}$ where $\mu_{0}=2 m_{t}$.

$$
\begin{gathered}
\sigma_{t \bar{t} t \bar{t}}^{\mathrm{LO}}\left(\mathrm{LHC}_{14 \mathrm{TeV}}, m_{t}=173.2 \mathrm{GeV}, \operatorname{MSTW} 2008 \mathrm{lo}\right)=12.056_{-4.876(-40 \%)}^{+9.364(+78 \%)} \mathrm{fb} \\
\sigma_{t \bar{t} t \bar{t}}^{\mathrm{NLO}}\left(\mathrm{LHC}_{14 \mathrm{TeV}}, m_{t}=173.2 \mathrm{GeV}, \text { MSTW2008nlo }\right)=15.33_{-3.81(-25 \%)}^{+3.95(+26 \%)} \mathrm{fb} .
\end{gathered}
$$

This leaves us with an NLO $\mathcal{K}$-factor equal to $\mathcal{K}=1.27$ and a positive NLO QCD correction of the order of $27 \%$. Also given in Table 2 are the integrated NLO cross sections for two values of the unphysical cutoff parameter $\alpha_{\max }$ that is a common modification of subtraction terms in the phase space away from the singularity, first introduced in [58]. To be more specific we use two values, $\alpha_{\max }=1$ that corresponds to the original formulation of $[56,57]$, and $\alpha_{\max }=0.01$. The independence of the final result on the value of the $\alpha_{\max }$ parameter is clearly visible in the Table 2 . This is a strong consistency check of the calculation of the real emission part. For more details on the $\alpha_{\max }$ implementation in the HelaC-Dipoles package see e.g. [22, 43].

The theoretical uncertainty of the total cross section, associated with neglected higher order terms in the perturbative expansion, can be estimated by varying the renormalization and factorization scales in $\alpha_{s}$ and PDFs, up and down by a factor 2 around the central scale of the process, i.e. $\mu_{0}$. An observed change in the value of $\sigma_{t \bar{t} t \bar{t}}^{\mathrm{LO}}$ for this two scale choices i.e. $0.5 \mu_{0}$ and $2 \mu_{0}$ is truly asymmetric. Taken very conservatively, as a maximum of these two results, the scale uncertainty at LO is estimated to be at the level of $78 \%$. However, in case like this it is more appropriate to symmetrize the errors. After symmetrization the scale uncertainty at LO is assessed to be instead of the order of $59 \%$. After inclusion of the NLO QCD corrections, the scale uncertainty is reduced down to 26\%. In Figure 4 a graphical presentation of the scale dependence is given, both at the LO and NLO. We 
observe a dramatic reduction of the scale uncertainty while going from LO to NLO. Figure 4 also shows the scale dependence of the $\mathrm{LO}$ cross section with the individual contributions of the two partonic channels. At the central scale, $\mu_{0}=2 m_{t}$, the $g g$ channel dominates the total $p p$ cross section by about $87 \%$ followed by the $q \bar{q}$ channel with about $13 \%$.

The theoretical uncertainty as obtained from the scale dependence of the cross section is not the only source of systematic uncertainties. Another source of uncertainties comes from the parameterization of PDFs. We estimate PDF uncertainties via the so called Hessian method where, besides the best fit PDFs, a set of 40 PDF parameterizations is provided that describes $\pm 1 \sigma$ variation of all parameters which have been used to obtain the global fit. These uncertainties are due to experimental errors in the various data that are used in the fits. We adopt the prescription from Ref. [61, 62] and based on these 40 sets calculate asymmetric uncertainties that estimate maximal variations of the result calculated for the central value in the positive and negative directions. They amount to $+5.7 \%$ and $-4.5 \%$. However, this method does not account for the theoretical assumptions that enter into parameterization of the PDFs which are difficult to quantify within a given scheme. Therefore, in the next step we use a different PDF set, namely CTEQ PDF [63, 64] that should have different theoretical assumptions. Specifically, we employ CT09MC1 PDFs with 1-loop running $\alpha_{s}$ at LO and CT10 PDFs with 2-loop running $\alpha_{s}$ at NLO. We compare the results for the central value using the best fit PDFs. With $\mu_{0}=2 m_{t}$ our findings can be summarized as follows:

$$
\begin{gathered}
\sigma_{t \bar{t} t \bar{t}}^{\mathrm{LO}}\left(\mathrm{LHC}_{14 \mathrm{TeV}}, m_{t}=173.2 \mathrm{GeV}, \mathrm{CT} 09 \mathrm{MC} 1\right)=11.414(8) \mathrm{fb}, \\
\sigma_{t \bar{t} t \bar{t}}^{\mathrm{NLO}}\left(\mathrm{LHC}_{14 \mathrm{TeV}}, m_{t}=173.2 \mathrm{GeV}, \mathrm{CT} 10\right)=14.37(2) \mathrm{fb} .
\end{gathered}
$$

The MSTW2008 results are larger than the CTEQ predictions by $5.6 \%$ at LO and $6.7 \%$ at NLO, which is comparable to the individual estimates of MSTW2008 PDF systematics ${ }^{2}$. In fact, since different values of $\alpha_{s}$ are associated with the MSTW2008 and the CTEQ sets one should also consider the uncertainties coming from the determination of the value of the strong coupling constant, which is fitted together with the PDFs. Clearly, further studies are needed to clarify these issues. However, since the PDF uncertainties for the process under scrutiny are well below the theoretical uncertainties due to scale dependence, which remain the dominant source of the theoretical systematics, we leave such studies for the future.

\subsection{Differential cross sections for $\mu_{0}=2 m_{t}$}

As already mentioned, total cross sections are mostly influenced by final-state production relatively close to the threshold as defined by particle masses. On the other hand, differential cross sections extend themselves up to energy scales that are much larger than the threshold, and may show larger shape distortions in such high-energy regions. Therefore, in the next step we turn our attention to the differential cross sections.

\footnotetext{
${ }^{2}$ Let us point out here that for the old CTEQ6 PDF sets $[65,66]$ that are still widely used in the phenomenological studies at the $\mathrm{LHC}$ we have obtained the following results $\sigma_{t \bar{t} t \bar{t}}^{\mathrm{LO}}\left(\mathrm{LHC}_{14 \mathrm{TeV}}, m_{t}=\right.$ 173.2 $\mathrm{GeV}, \mathrm{CTEQ} 6 \mathrm{~L} 1)=8.259(4) \mathrm{fb}, \sigma_{t \bar{t} t \bar{t}}^{\mathrm{NLO}}\left(\mathrm{LHC}_{14 \mathrm{TeV}}, m_{t}=173.2 \mathrm{GeV}, \mathrm{CTEQ} 6 \mathrm{M}\right)=14.74(2) \mathrm{fb}$.
} 

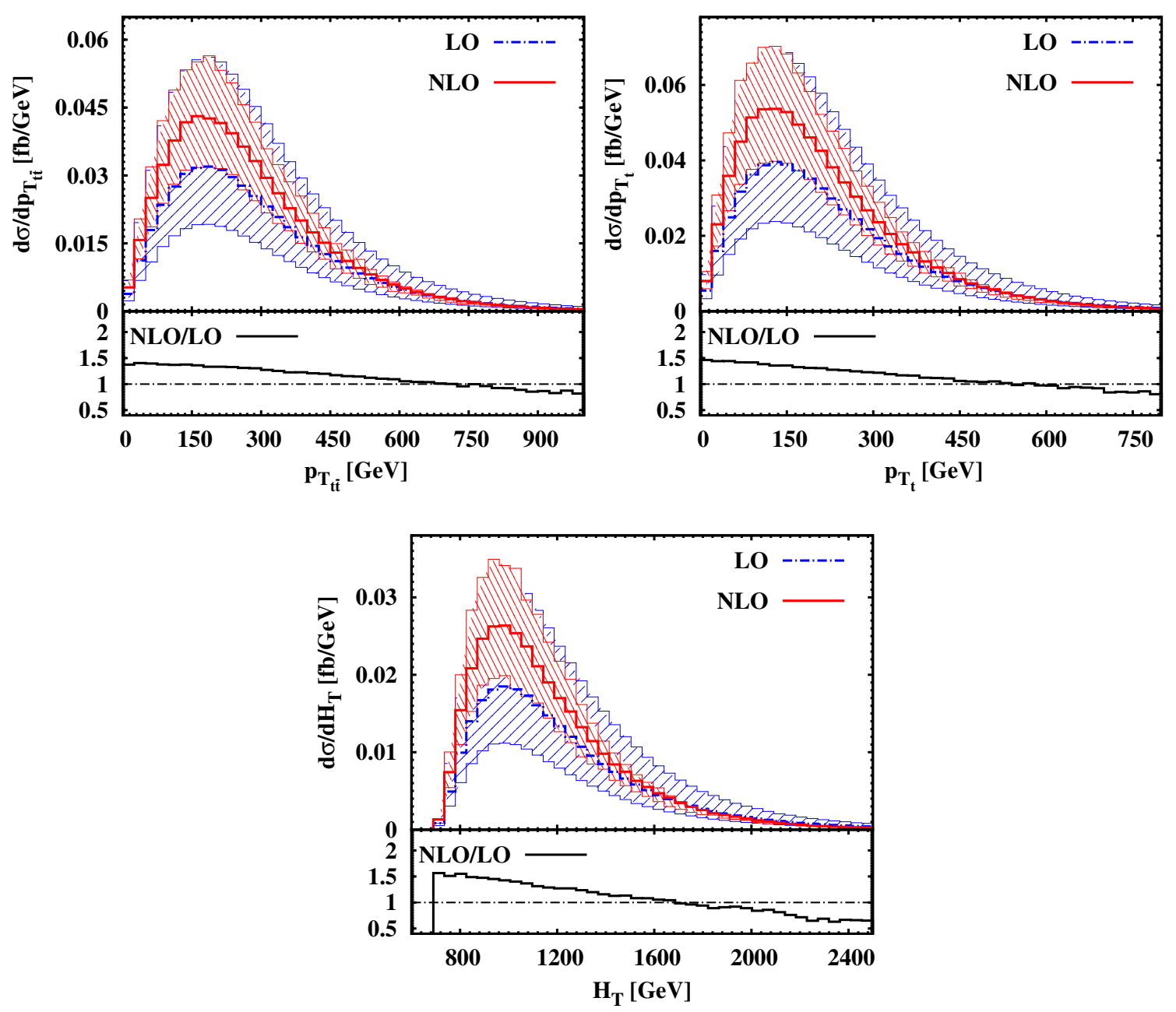

Figure 5. Averaged differential cross section distributions as a function of transverse momentum of the $t \bar{t}$ pair (upper-left panel) and the top quark (upper-right panel) for $p p \rightarrow t \bar{t} t \bar{t}+X$ production at the LHC with $\sqrt{s}=14 \mathrm{TeV}$. Also shown is the differential cross section distribution as a function of the total transverse energy of the system (lower panel). The dash-dotted (blue) curve corresponds to the LO, whereas the solid (red) one to the $N L O$ result. The scale choice is $\mu_{F}=\mu_{R}=\mu_{0}=2 m_{t}$. The uncertainty bands depict scale variation. The lower panels display the differential $\mathcal{K}$ factor.

We have checked as many as 16 observables. Here we present three cases where the differential $\mathcal{K}$ factor, defined as the bin-by-bin ratio of the NLO result to the $\mathrm{LO}$ one for the central scale value $\mu_{0}$, has been found to be mostly distorted. In Figure 5 we present the averaged transverse momentum distribution of $t \bar{t}$ pair and of the top quark together with the distribution of the total transverse energy of the $t \bar{t} t \bar{t}$ system. The dash-dotted (blue) curve corresponds to the LO, whereas the solid (red) one to the NLO result. The upper panels show the distributions themselves and additionally include the scale-dependence bands obtained with a variation of the central scale by a factor of two. The lower panels display the differential $\mathcal{K}$ factor. 
We observe that at the LHC, employing a fixed scale $\mu_{0}=2 m_{t}$, the NLO corrections to the transverse momentum distributions do not simply rescale the LO shapes, but also induce distortions at the level of $60 \%$. In case of the total transverse energy of the system, which we define as a sum of (anti-)top quark transverse energies

$$
\begin{gathered}
H_{T}=\sum_{i=1,2} E_{T}\left(t_{i}\right)+\sum_{i=1,2} E_{T}\left(\bar{t}_{i}\right), \\
E_{T}(t)=\sqrt{m_{t}^{2}+p_{T}^{2}(t)},
\end{gathered}
$$

the situation is even more severe with an observed distortion at the level of $80 \%$ or more. Clearly, large and negative NLO corrections affect the high $H_{T}$ region. One can also note that the NLO error bands, estimated through scale variation, do not fit nicely within the LO ones at low $p_{T}$ 's, as one should expect from a well-behaved perturbative expansion. We can summarize our conclusions at this stage by remarking that the fixed-scale choice $\mu_{R}=$ $\mu_{F}=\mu_{0}=2 m_{t}$ does not ensure stable shapes when going from LO to NLO. Therefore, differential cross sections are properly described only when the NLO QCD corrections are taken into account.

\subsection{Integrated cross section and its scale dependence for $\mu_{0}=H_{T} / 4$}

With the goal of stabilizing shapes in the high $p_{T}$ and $H_{T}$ regions, that are relevant for the new physics searches, we have explored a dynamical choice for $\mu_{R}$ and $\mu_{F}$ that helps to achieve flatter differential $\mathcal{K}$-factors, thus describing more appropriately the kinematics of the process far away from the threshold. To this end, we have incorporated the dynamical scale option into the HELAC-NLO framework. The implementation has been carefully crosschecked by reproducing partial and total cross section results for the NLO QCD corrections to the $p p \rightarrow t \bar{t} b \bar{b}+X$ process with a dynamical scale for the setup number I as given in Ref. [21]. A per-mille level agreement has been found in all cases.

For the process at hand, we explored several possibilities and decided in the end to consider the dynamical scale $\mu_{R}=\mu_{F}=\mu_{0}=H_{T} / 4^{3}$. While preserving moderate NLO QCD corrections and the dramatic reduction of the theoretical uncertainty, this new scale choice turns out to be particularly effective in improving the stability of distribution shapes.

Table 3 shows the integrated cross sections at LO and NLO for $p p \rightarrow t \bar{t} t \bar{t}+X$ production at the LHC with $\sqrt{s}=14 \mathrm{TeV}$, using the same settings as before with the only exception of the scale choice, this time set to be $\mu_{0}=H_{T} / 4$. Our results can be summarized as follows:

$$
\begin{aligned}
& \sigma_{t \bar{t} t \bar{t}}^{\mathrm{LO}}\left(\mathrm{LHC}_{14 \mathrm{TeV}}, m_{t}=173.2 \mathrm{GeV}, \operatorname{MSTW} 2008 \mathrm{lo}\right)=13.891_{-5.711(-41 \%)}^{+11.074(+80 \%)} \mathrm{fb}, \\
& \sigma_{t \bar{t} t \bar{t}}^{\mathrm{NLO}}\left(\mathrm{LHC}_{14 \mathrm{TeV}}, m_{t}=173.2 \mathrm{GeV}, \text { MSTW2008nlo }\right)=16.87_{-4.26(-25 \%)}^{+4.04(+24 \%)} \mathrm{fb} .
\end{aligned}
$$

The new results are a bit higher, i.e. by $15 \%$ at LO and by $10 \%$ at NLO compared with Table 2, which is perfectly within theoretical error estimates at the corresponding

\footnotetext{
${ }^{3}$ The sum of the transverse energies of massless outgoing partons and leptons, has already been advocated as a good scale choice in the study of NLO QCD corrections to the differential distributions for $p p \rightarrow V+3 j$ process, where $V=W^{ \pm}, Z / \gamma^{\star}[34,35]$.
} 

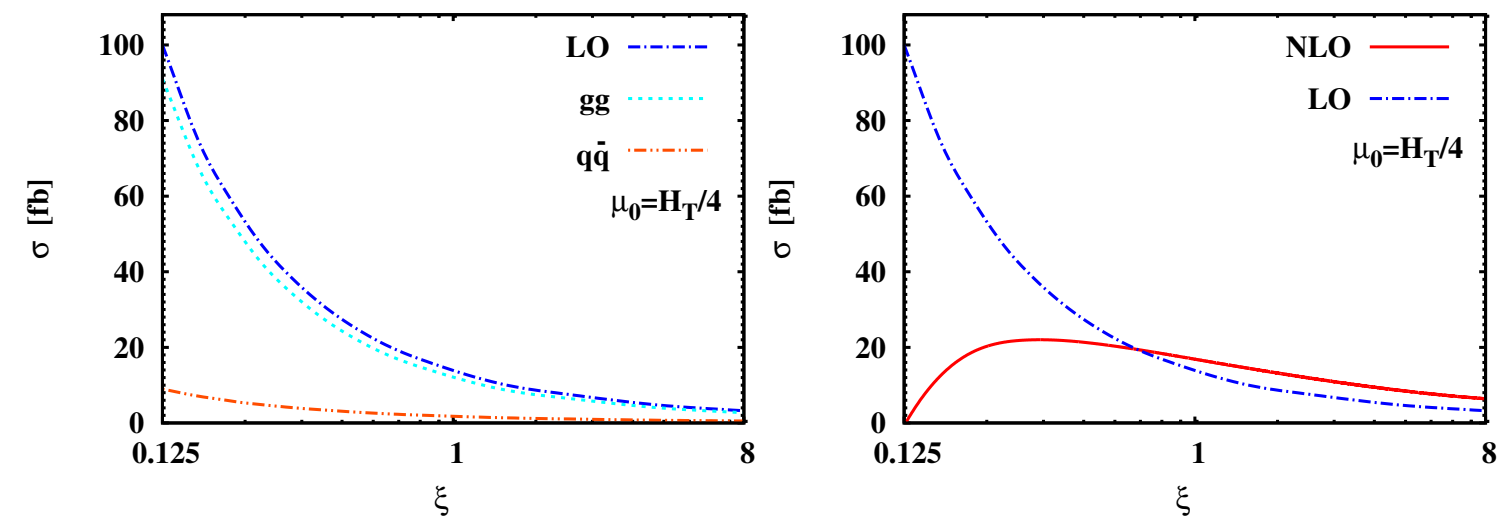

Figure 6. Scale dependence of the LO cross section with the individual contributions of the partonic channels (left panel) and scale dependence of the LO and NLO cross sections (right panel) for the $p p \rightarrow t \bar{t} t \bar{t}+X$ process at the LHC for $\sqrt{s}=14$ TeV. The scale is set to a common value $\mu_{R}=\mu_{F}=\xi \cdot \mu_{0}$ where $\mu_{0}=H_{T} / 4$.

\begin{tabular}{||c|c|c|c|c|c||}
\hline \hline PROCESS & $\sigma_{\mathrm{LO}}[\mathrm{fb}]$ & $\sigma_{\mathrm{NLO}}^{\alpha_{\max }=1}[\mathrm{fb}]$ & $\sigma_{\mathrm{NLO}}^{\alpha_{\max }=0.01}[\mathrm{fb}]$ & K-FACTOR & {$[\%]$} \\
\hline \hline$p p \rightarrow t \bar{t} t \bar{t}+X$ & $13.891(9)$ & $16.87(2)$ & $16.86(3)$ & 1.21 & 21 \\
\hline \hline
\end{tabular}

Table 3. Integrated cross section at LO and NLO for $p p \rightarrow t \bar{t} t \bar{t}+X$ production at the LHC with $\sqrt{s}=14 \mathrm{TeV}$. Results for the MSTW2008 PDF set are presented. In the last two columns the K factor, defined as the ratio of the NLO cross section to the respective $L O$ result, and NLO corrections in $\%$ are given. The scale choice is $\mu_{F}=\mu_{R}=\mu_{0}=H_{T} / 4$.

perturbative order level. Moreover, the $\mathcal{K}$-factor obtained with this new scale is smaller, of the order of $\mathcal{K}=1.21$. The independence of the final result on the value of the $\alpha_{\max }$ parameter has also been checked in this case as shown in Table 3. For completeness, we show in Figure 6 the scale dependence of the LO and NLO cross sections. Again we observe a striking reduction of the scale uncertainty while going from LO to NLO. Varying the scale up and down by a factor 2 changes the cross section by $+80 \%$ and $-41 \%$ in the LO case, whereas in the NLO case we obtain a variation of $+24 \%$ and $-25 \%$. With the evaluation of NLO QCD corrections the theoretical error has been decreased from about $80 \%(60 \%)$ down to $25 \%$.

\subsection{Differential cross sections for $\mu_{0}=H_{T} / 4$}

As desired, the results for the integrated cross sections have only slightly changed in comparison with the fixed-scale case. In the following, we study the impact of the different scale choice on the differential cross sections. In Figure 7 the averaged differential distribution 

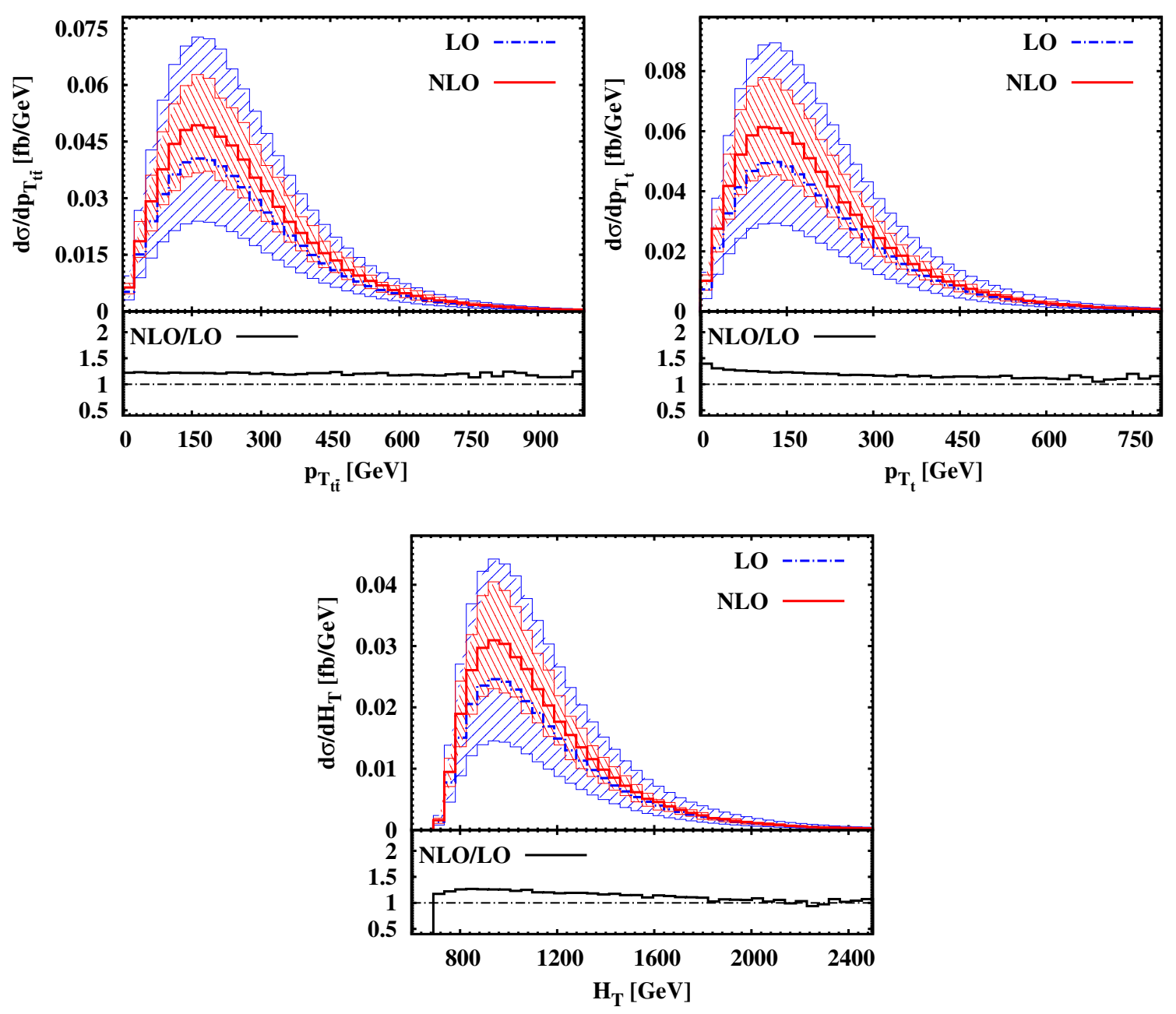

Figure 7. Averaged differential cross section distributions as a function of transverse momentum of the $t \bar{t}$ pair (upper-left panel) and the top quark (upper-right panel) for $p p \rightarrow t \bar{t} t \bar{t}+X$ production at the LHC with $\sqrt{s}=14 \mathrm{TeV}$. Also shown is the differential cross section distribution as a function of the total transverse energy of the system (lower panel). The dash-dotted (blue) curve corresponds to the LO, whereas the solid (red) one to the $N L O$ result. The scale choice is $\mu_{F}=\mu_{R}=\mu_{0}=H_{T} / 4$. The uncertainty bands depict scale variation. The lower panels display the differential $\mathcal{K}$ factor.

as a function of the transverse momentum of the $t \bar{t}$ pair and the top quark together with total transverse energy are shown once again, however, this time with the renormalization and factorization scales calculated on an event-by-event basis. Instead of $60 \%$ distortions that we have obtained in the previous case for the $p_{T_{t \bar{t}}}$ and $p_{T_{t}}$ distributions, the moderate and positive corrections of the order or $20 \%$ have been established over the whole range of $p_{T}$. An improvement in the differential $\mathcal{K}$-factor is clearly visible in both cases. The same conclusions can be drawn for the $H_{T}$ distribution. In this case the improvement is even more impressive. In addition, one can observe that the NLO error bands as calculated through scale variation nicely fit within the LO error bands. 

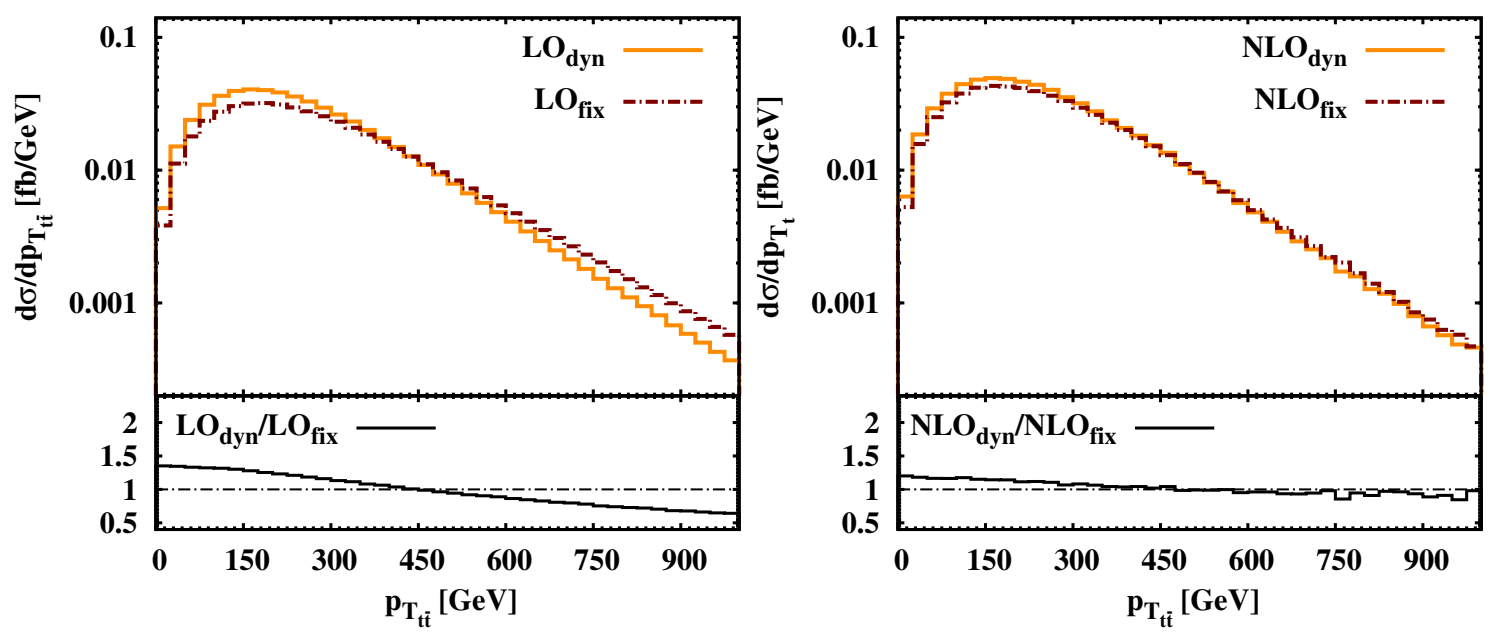

Figure 8. Averaged differential cross section distributions as a function of transverse momentum of the $t \bar{t}$ pair at $L O$ (left panel) and at $N L O$ (right panel) for $p p \rightarrow t \bar{t} t \bar{t}+X$ production at the $L H C$ with $\sqrt{s}=14 \mathrm{TeV}$. The dash-dotted (orange) curve corresponds to $\mu_{0}=2 m_{t}$, whereas the dashed (brown) one to $\mu_{0}=H_{T} / 4$. The lower panels display the ratio of the result with the dynamic scale versus the fixed scale.
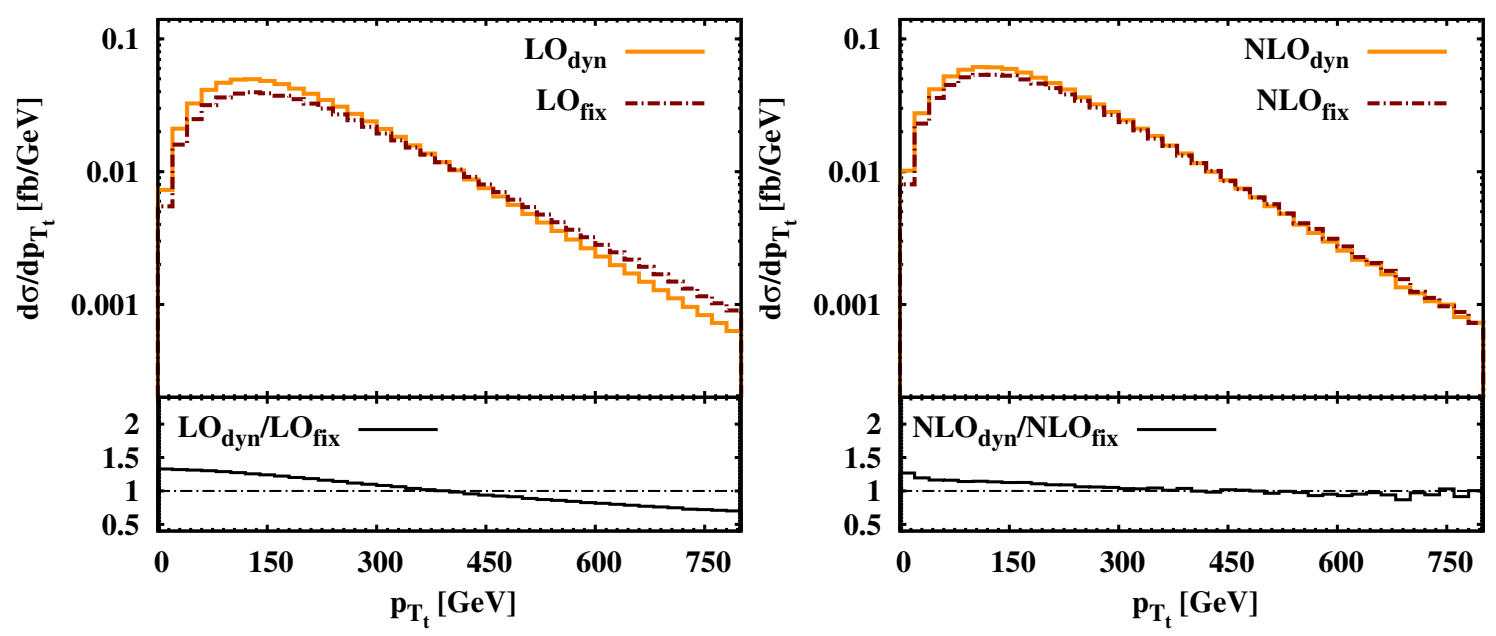

Figure 9. Averaged differential cross section distributions as a function of transverse momentum of the top quark at $L O$ (left panel) and at $N L O$ (right panel) for $p p \rightarrow t \bar{t} t \bar{t}+X$ production at the LHC with $\sqrt{s}=14 \mathrm{TeV}$. The dash-dotted (orange) curve corresponds to $\mu_{0}=2 m_{t}$, whereas the dashed (brown) one to $\mu_{0}=H_{T} / 4$. The lower panels display the ratio of the result with the dynamic scale versus the fixed scale.

In general, the idea behind a dynamical scale is to accommodate for multiscale kinematics. In fact, we are not trying to reduce the $\mathcal{K}$-factor as defined by the ratio of total cross sections, but rather to obtain a constant one for the distributions. The fixed order approximation is meaningful, when the improved scale choice affects NLO cross sections 

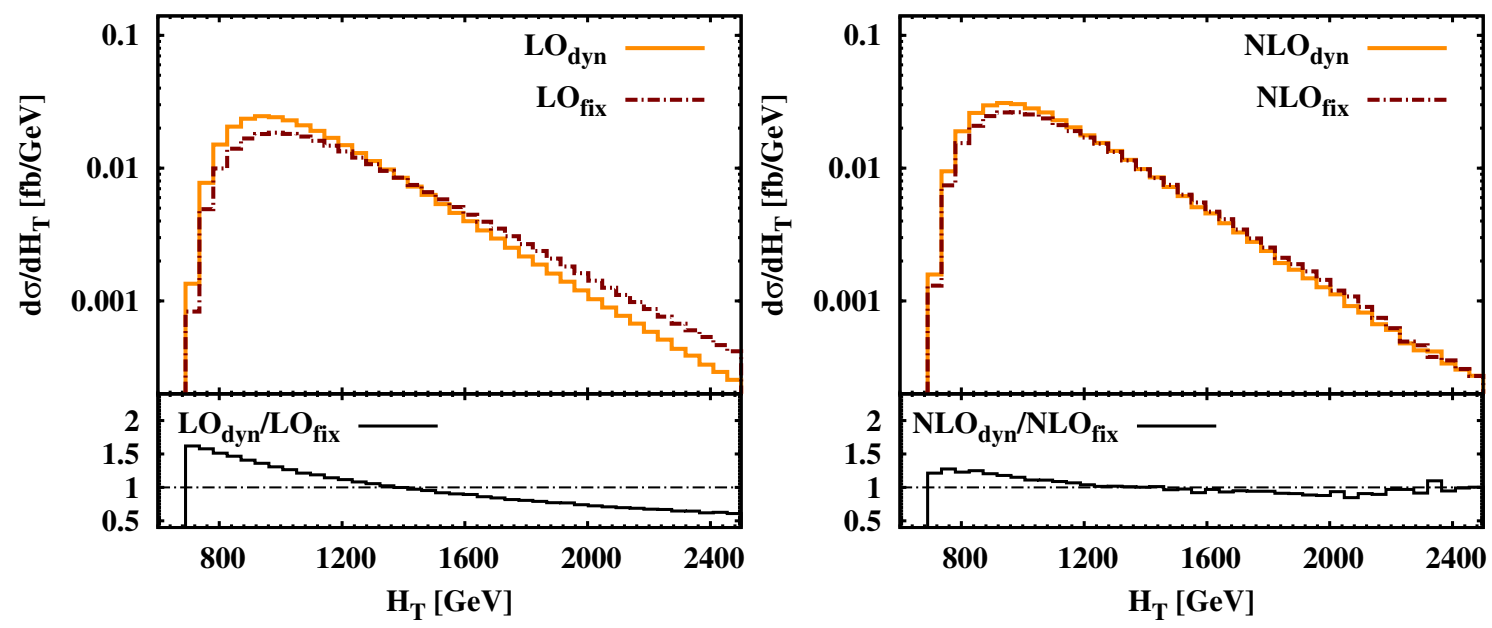

Figure 10. Differential cross section distributions as a function of total transverse energy of the $t \bar{t} t \bar{t}$ system at $L O$ (left panel) and at $N L O$ (right panel) for $p p \rightarrow t \bar{t} t \bar{t}+X$ production at the $L H C$ with $\sqrt{s}=14 \mathrm{TeV}$. The dash-dotted (orange) curve corresponds to $\mu_{0}=2 m_{t}$, whereas the dashed (brown) one to $\mu_{0}=H_{T} / 4$. The lower panels display the ratio of the result with the dynamic scale versus the fixed scale.
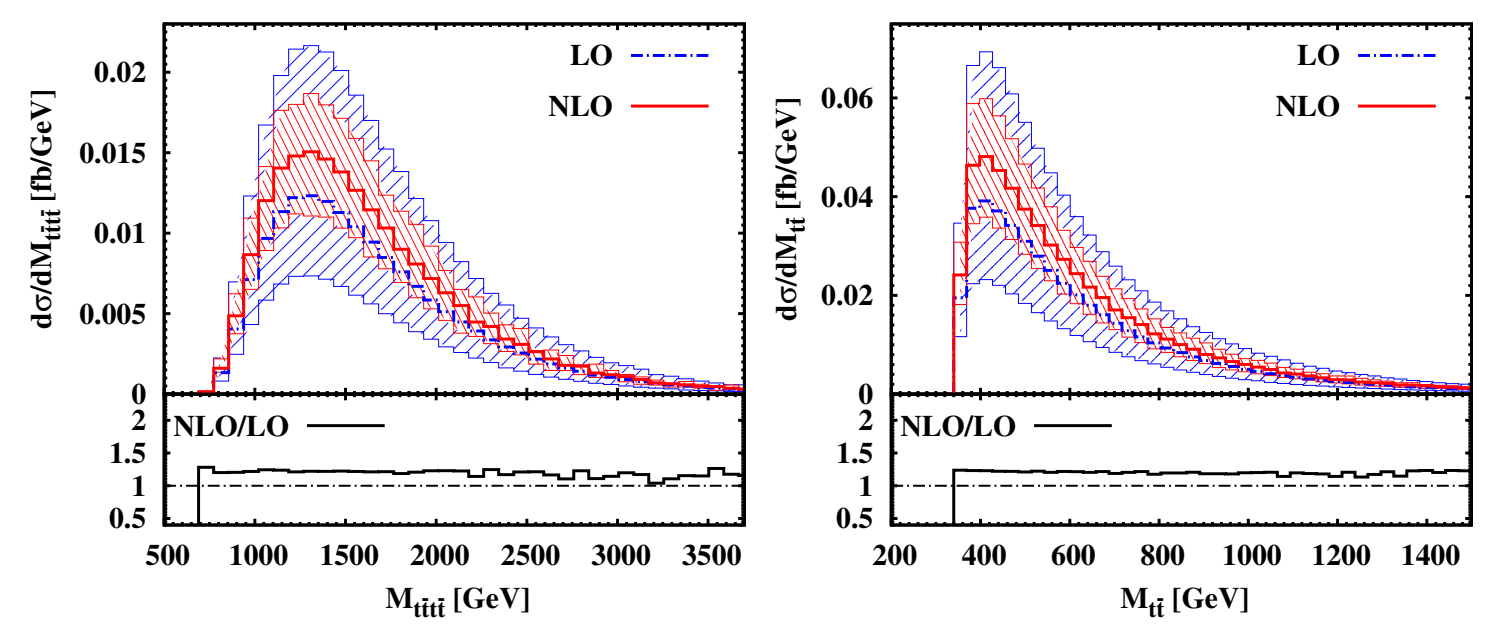

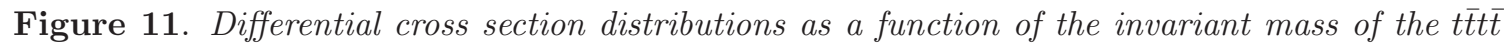
system (left panel) and the t $\bar{t}$ pair (right panel) for $p p \rightarrow t \bar{t} t \bar{t}+X$ production at the LHC with $\sqrt{s}=14 \mathrm{TeV}$. The dash-dotted (blue) curve corresponds to the LO, whereas the solid (red) one to the $N L O$ result. The scale choice is $\mu_{F}=\mu_{R}=H_{T} / 4$. The uncertainty bands depict scale variation. The lower panels display the differential $\mathcal{K}$ factor.

to a much lower extent than the LO ones. We also demonstrate that with a proper scale, we can already obtain good results with a constant rescaling of LO distributions. There always remains the question of how these ideas work in practice. A convincing example is illustrated on Figure 8 - Figure 10, for three different observables, where fixed scale $\mathcal{K}$-factors were most unsatisfactory. With the luxury of an NLO cross section, the im- 

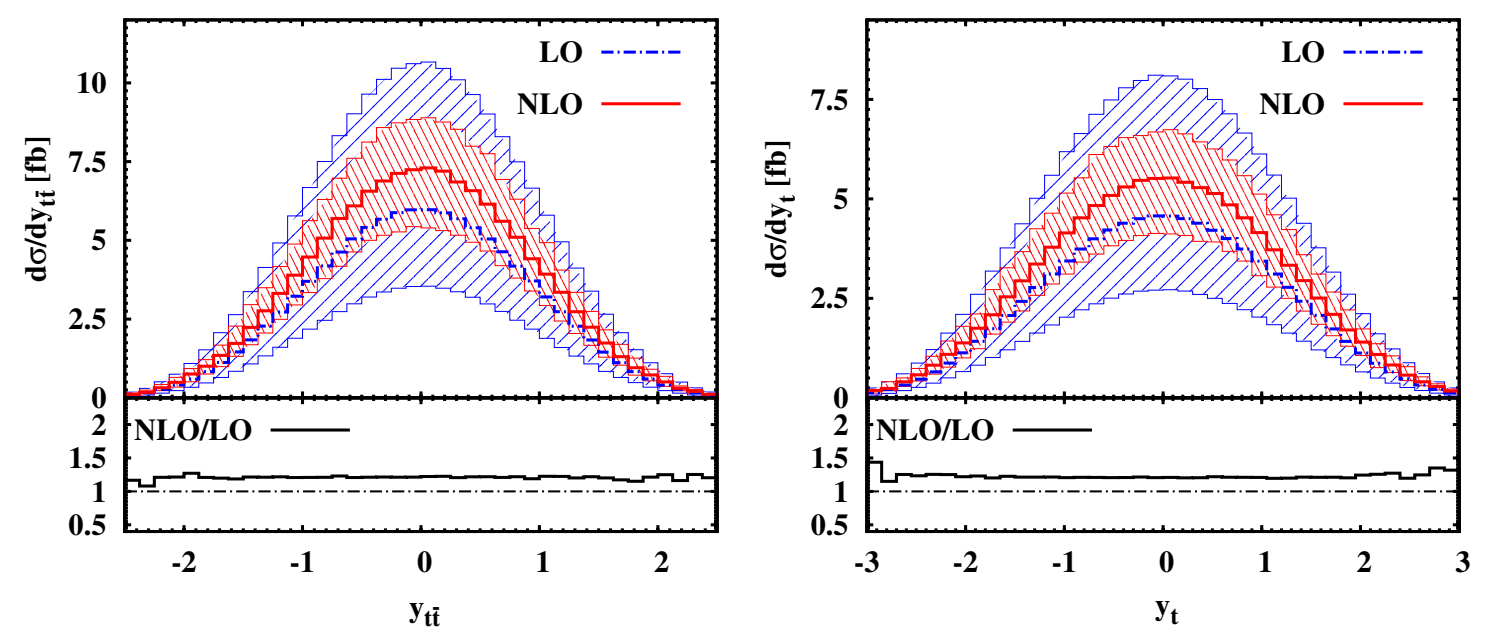

Figure 12. Averaged differential cross section distributions as a function of rapidity of the t $\bar{t}$ pair (left panel) and the top quark (right panel) for $p p \rightarrow t \bar{t} t \bar{t}+X$ production at the LHC with $\sqrt{s}=14 \mathrm{TeV}$. The dash-dotted (blue) curve corresponds to the LO, whereas the solid (red) one to the $N L O$ result. The scale choice is $\mu_{F}=\mu_{R}=\mu_{0}=H_{T} / 4$. The uncertainty bands depict scale variation. The lower panels display the differential $\mathcal{K}$ factor.

provement obtained with a dynamic scale, which can only be guessed at for the lack of a NNLO result, is moderate. The change in the shape of LO distributions is, on the other hand, rather strong.

We have compared these two scale choices for many other observables and in all cases the new, dynamic one has decreased the shape difference of distributions while going from LO to NLO. As an example, the invariant mass distribution of the $t \bar{t} t \bar{t}$ systems and the averaged invariant mass distribution of the $t \bar{t}$ pair are illustrated in Figure 11. Even though the invariant mass distributions peak strongly at the corresponding thresholds, there are non-negligible tails that are extended to very high invariant mass values. Also for these kinematical regions an almost flat differential $\mathcal{K}$-factor has been obtained. In addition, in Figure 12, the angular differential distributions are presented. We show in particular the averaged rapidity distribution of the $t \bar{t}$ pair and the averaged rapidity distribution of the top quark. As one can see from Figure 12, the $t \bar{t}$ pairs and the top quarks are predominantly produced in the central region. Also here, the differential $\mathcal{K}$-factor is near constant within the whole range of $y$.

Through the implementation of the dynamical scale large discrepancies between shapes of distributions at NLO and LO have disappeared. New differential $\mathcal{K}$-factors suggest that the proper scale choice in LO calculations, which describes the kinematics of the whole process on an event-by-even basis, together with a suitably chosen global $\mathcal{K}$-factor would be sufficient for this process. This is good news taking into account that the $\mathrm{LO}$ calculations are not only less costly, but can be easily merged via CKKW/MLM procedures [67] with parton shower programs to obtain complete inclusive hadron level events samples that can be directly compared with the experimental data. 

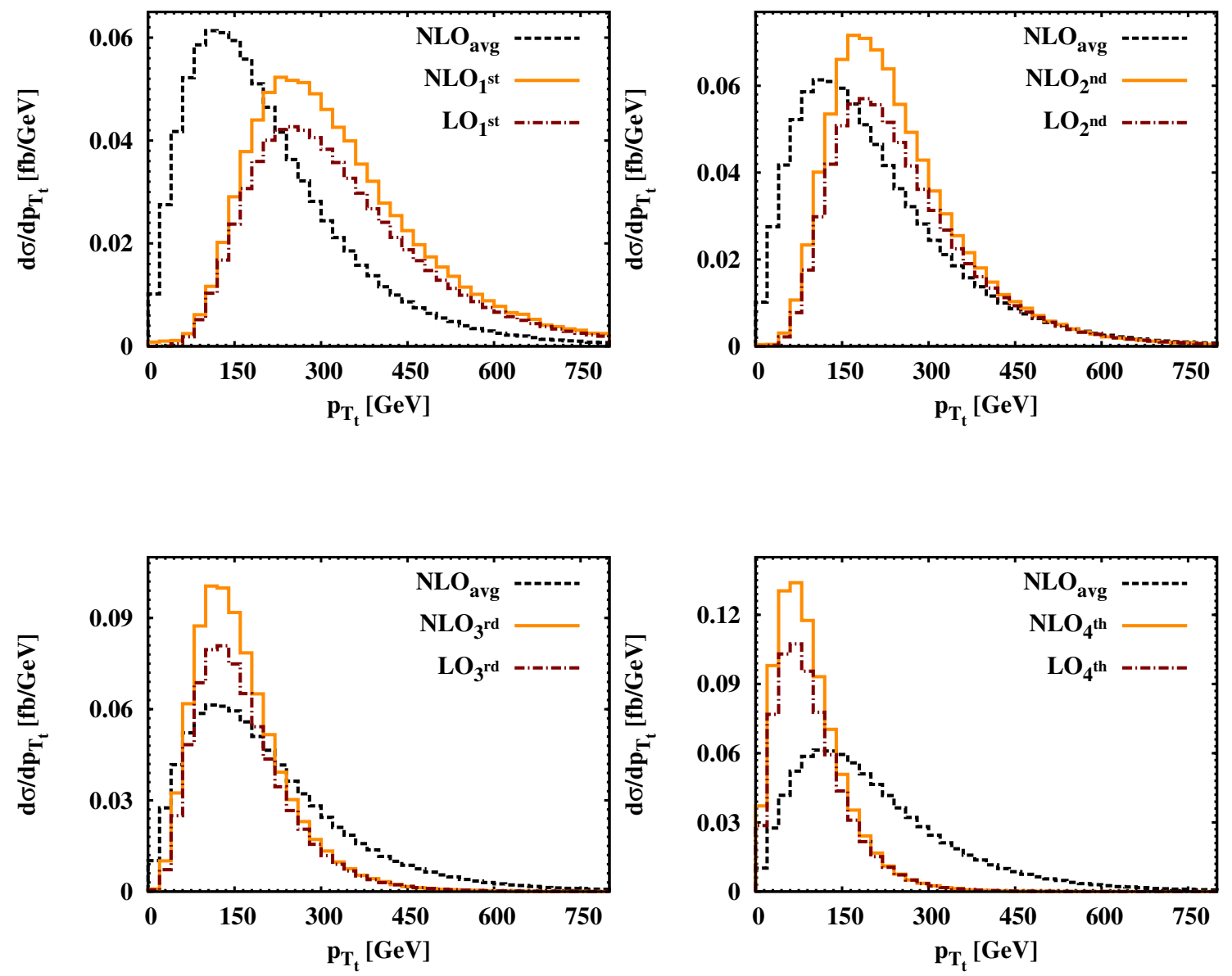

Figure 13. Differential cross section distributions as a function of the transverse momentum of the 1st, 2nd, 3rd and the 4th hardest top quark at LO and NLO for $p p \rightarrow t \bar{t} t \bar{t}+X$ production at the LHC with $\sqrt{s}=14 \mathrm{TeV}$. The scale choice is $\mu_{F}=\mu_{R}=H_{T} / 4$. Also shown is the averaged transverse momentum of the top quark at NLO.

Once the impact of the NLO QCD corrections to the differential cross sections has been established, we turn our attention to the properties of the top quarks. In Figure 13 a comparison of the transverse momentum spectra of the first, second, third and the forth hardest top quark in the $p p \rightarrow t \bar{t} t \bar{t}+X$ production at LO and NLO is displayed. In each plot, the averaged transverse momentum of the top quark at NLO is also shown as a benchmark. As expected, the shape of the distributions and their peak change when moving from the hardest to the softest top quark configurations.

Finally, the invariant mass of the two top quarks with the highest $p_{T}$ is given in Figure 14. This observable is particular interesting in view of new physics searches where the mass of a new heavy resonance that decays into the top-anti-top pair is reconstructed as 

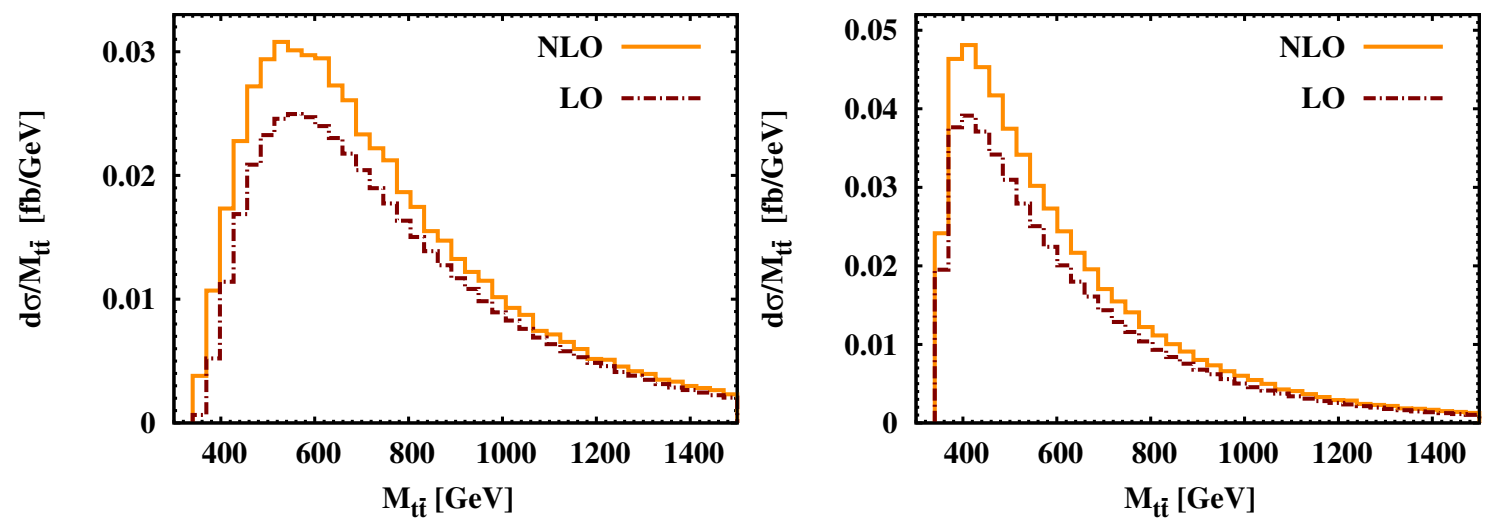

Figure 14. Differential cross section distributions as a function of the invariant mass of the t $\bar{t}$ pair at LO and NLO for $\sqrt{s}=14 \mathrm{TeV}$. Left panel: the invariant mass of the two top quarks with the highest $p_{T}$. Right panel: the averaged invariant mass of the $t \bar{t}$ pair. The scale choice is $\mu_{F}=\mu_{R}=H_{T} / 4$.

the invariant mass of the two objects with the highest $p_{T}$ in the event. For comparison, the averaged invariant mass of the $t \bar{t}$ pair is also presented.

\section{Summary and Conclusions}

In this paper we have presented a computation of the NLO QCD corrections to four top quark production at the LHC. The total cross section and its scale dependence have been evaluated for two different scale choices, i.e. for the fixed scale $\mu_{R}=\mu_{F}=\mu_{0}=2 m_{t}$ and for the dynamical scale $\mu_{R}=\mu_{F}=\mu_{0}=H_{T} / 4$. The impact of the NLO QCD corrections on the integrated cross sections is moderate, of the order of $27 \%$ for $\mu_{0}=2 m_{t}$ and $21 \%$ for $\mu_{0}=H_{T} / 4$. As to the theoretical uncertainty of our calculation, the contribution related to unknown higher-order corrections, as obtained by studying the scale dependence of our NLO predictions, is of the order of $25 \%$. We have also analyzed the theoretical error arising from different parametrizations of PDFs, being able to quantify it at the level of $5 \%-6 \%$, thus well below the uncertainty associated with scale dependence.

Looking only at the total cross section, which is mostly influenced by final state production relatively close to the threshold, both scale choices are in equally good shape and the results agree well within the corresponding theoretical errors. On the other hand, differential cross sections show large differences in shape, with distortions up to $80 \%$ observed within our fixed-scale setting. In particular, large negative corrections are clearly visible in the tails of several distributions. Thus, an accurate description of the shapes of observables can be given only via full NLO QCD computation in this case. Instead, adopting our dynamical scale choice, results have moderate, positive and almost constant corrections of the order of $20 \%$ for all the investigated observables. This fact suggests that the proposed 
dynamical scale efficiently accommodates for the multiscale kinematics of the process.

Well-behaved as it is, the proposed dynamical scale has a number of advantages for phenomenological studies. Indeed, it can be used within a LO calculation, together with a suitably chosen global $\mathcal{K}$-factor, to obtain results that well approximate the full NLO QCD calculation and can be merged with parton shower programs to obtain realistic hadronic events, directly comparable with the experimental data for new physics searches. Particularly interesting observables in this sense are the invariant mass and the total transverse energy of the $t \bar{t} t \bar{t}$ system, together with the invariant mass of the two hardest top quarks.

As a final remark we point out that, despite its relatively small cross section, a good theoretical control over the SM $p p \rightarrow t \bar{t} t \bar{t}$ background can be phenomenologically relevant. Our NLO QCD predictions are of the order of $17 \pm 4$ [scales] \pm 1 [PDF] fb for $\sqrt{s}=14$ $\mathrm{TeV}$. For comparison, typical predictions of new physics scenarios such as effective fourtop interactions, Kaluza-Klein gluons or the so-called top-philic $Z^{\prime}$ (i.e., a $Z^{\prime}$ which couples to third-family quarks only) are set in the range $5-100 \mathrm{fb}$ for $m_{\text {new }}=1 \mathrm{TeV}$ and $1-20 \mathrm{fb}$ for $m_{\text {new }}=1.5 \mathrm{TeV}$, where $m_{\text {new }}$ is the mass of the new heavy particle or, more generally, the energy scale associated with new physics effects. For masses greater than $2-3 \mathrm{TeV}$ rates are below $1 \mathrm{fb}[8]$. We believe that a NLO analysis of $p p \rightarrow t \bar{t} t \bar{t}$ at the LHC is a necessary step towards a correct interpretation of the possible signals of new physics that may arise in this channel.

\section{Acknowledgments}

The calculations have been performed on the Grid Cluster of the Bergische Universität Wuppertal, financed by the Helmholtz - Alliance Physics at the Terascale and the BMBF.

The work of M.W. was supported by the Initiative and Networking Fund of the Helmholtz Association, contract HA-101 (Physics at the Terascale).

The work of G.B. was supported by the DFG Sonderforschungsbereich/Transregio 9 Computergestützte Theoretische Teilchenphysik.

We thank Ansgar Denner for providing us with partial results for the NLO QCD corrections to the $p p \rightarrow t \bar{t} b \bar{b}+X$ production with $\mu_{R}^{2}=\mu_{F}^{2}=\mu_{0}^{2}=m_{t} \sqrt{p_{T}(b) \cdot p_{T}(\bar{b})}$ that helped to cross check our implementation of the dynamical scale in the HELAC-NLO framework.

We also would like to thank Alberto Guffanti and Maria Ubiali for discussions concerning PDFs.

\section{References}

[1] D. A. Dicus, B. Dutta and S. Nandi, "Top quark signature in extended color theories", Phys. Rev. D 51 (1995) 6085, [hep-ph/9412370].

[2] A. Djouadi, G. Moreau and R. K. Singh, "Kaluza-Klein excitations of gauge bosons at the LHC", Nucl. Phys. B 797 (2008) 1, [arXiv:0706.4191 [hep-ph]].

[3] M. Guchait, F. Mahmoudi and K. Sridhar, "Associated production of a Kaluza-Klein excitation of a gluon with a $t$ anti-t pair at the LHC", Phys. Lett. B 666, 347 (2008), [arXiv:0710.2234 [hep-ph]]. 
[4] B. Lillie, J. Shu and T. M. P. Tait, "Top Compositeness at the Tevatron and LHC", JHEP 0804 (2008) 087, [arXiv:0712.3057 [hep-ph]].

[5] A. Pomarol and J. Serra, "Top Quark Compositeness: Feasibility and Implications", Phys. Rev. D 78 (2008) 074026, [arXiv:0806.3247 [hep-ph]].

[6] K. Kumar, T. M. P. Tait and R. Vega-Morales, "Manifestations of Top Compositeness at Colliders", JHEP 0905 (2009) 022, [arXiv:0901.3808 [hep-ph]].

[7] D. E. Morrissey, T. Plehn and T. M. P. Tait, "Physics searches at the LHC", Phys. Rept. 515 (2012) 1, [arXiv:0912.3259 [hep-ph]].

[8] G. Brooijmans et al. [New Physics Working Group Collaboration], "New Physics at the LHC. A Les Houches Report: Physics at TeV Colliders 2009 - New Physics Working Group", arXiv:1005.1229 [hep-ph].

[9] S. Jung and J. D. Wells, "Low-scale warped extra dimension and its predilection for multiple top quarks", JHEP 1011 (2010) 001, [arXiv:1008.0870 [hep-ph]].

[10] T. Gregoire, E. Katz and V. Sanz, "Four top quarks in extensions of the standard model", Phys. Rev. D 85 (2012) 055024, [arXiv:1101.1294 [hep-ph]].

[11] F. Gianotti, M. L. Mangano, T. Virdee, S. Abdullin, G. Azuelos, A. Ball, D. Barberis and A. Belyaev et al., "Physics potential and experimental challenges of the LHC luminosity upgrade”, Eur. Phys. J. C 39 (2005) 293, [hep-ph/0204087].

[12] U. Baur, T. Plehn and D. L. Rainwater, "Measuring the Higgs boson self coupling at the LHC and finite top mass matrix elements", Phys. Rev. Lett. 89 (2002) 151801, [hep-ph/0206024].

[13] M. Toharia and J. D. Wells, "Gluino decays with heavier scalar superpartners", JHEP 0602 (2006) 015, [hep-ph/0503175].

[14] D. Alves et al. [LHC New Physics Working Group Collaboration], "Simplified Models for LHC New Physics Searches", arXiv:1105.2838 [hep-ph].

[15] J. A. Maestre, S. Alioli, J. R. Andersen, R. D. Ball, A. Buckley, M. Cacciari, F. Campanario and N. Chanon et al., "The SM and NLO Multileg and SM MC Working Groups: Summary Report", [arXiv:1203.6803 [hep-ph]].

[16] Z. Bern, L. J. Dixon, D. C. Dunbar and D. A. Kosower, "One loop n point gauge theory amplitudes, unitarity and collinear limits”, Nucl. Phys. B 425 (1994) 217, [hep-ph/9403226].

[17] R. Britto, F. Cachazo and B. Feng, "Generalized unitarity and one-loop amplitudes in N=4 super-Yang-Mills", Nucl. Phys. B 725 (2005) 275, [hep-th/0412103].

[18] G. Ossola, C. G. Papadopoulos and R. Pittau, "Reducing full one-loop amplitudes to scalar integrals at the integrand level", Nucl. Phys. B 763 (2007) 147, [hep-ph/0609007].

[19] W. T. Giele, Z. Kunszt and K. Melnikov, "Full one-loop amplitudes from tree amplitudes", JHEP 0804 (2008) 049, [arXiv:0801.2237 [hep-ph]].

[20] A. Bredenstein, A. Denner, S. Dittmaier and S. Pozzorini, "NLO QCD corrections to $p p \rightarrow t$ anti-t b anti-b + X at the LHC", Phys. Rev. Lett. 103 (2009) 012002, [arXiv:0905.0110 [hep-ph]].

[21] A. Bredenstein, A. Denner, S. Dittmaier and S. Pozzorini, "NLO QCD Corrections to Top Anti-Top Bottom Anti-Bottom Production at the LHC: 2. full hadronic results", JHEP 1003 (2010) 021, [arXiv:1001.4006 [hep-ph]]. 
[22] G. Bevilacqua, M. Czakon, C. G. Papadopoulos, R. Pittau and M. Worek, "Assault on the NLO Wishlist: $p p \rightarrow t$ anti-t b anti-b”, JHEP 0909 (2009) 109, [arXiv:0907.4723 [hep-ph]].

[23] M. Worek, "On the next-to-leading order QCD K-factor for top anti-top bottom anti-bottom production at the TeVatron", JHEP 1202 (2012) 043, [arXiv:1112.4325 [hep-ph]].

[24] G. Bevilacqua, M. Czakon, C. G. Papadopoulos and M. Worek, "Dominant QCD Backgrounds in Higgs Boson Analyses at the LHC: A Study of $p p \rightarrow t$ anti- +2 jets at Next-To-Leading Order", Phys. Rev. Lett. 104 (2010) 162002, [arXiv:1002.4009 [hep-ph]].

[25] G. Bevilacqua, M. Czakon, C. G. Papadopoulos and M. Worek, "Hadronic top-quark pair production in association with two jets at Next-to-Leading Order QCD", Phys. Rev. D 84 (2011) 114017, [arXiv:1108.2851 [hep-ph]].

[26] G. Bevilacqua, M. Czakon, A. van Hameren, C. G. Papadopoulos and M. Worek, "Complete off-shell effects in top quark pair hadroproduction with leptonic decay at next-to-leading order", JHEP 1102 (2011) 083, [arXiv:1012.4230 [hep-ph]].

[27] A. Denner, S. Dittmaier, S. Kallweit and S. Pozzorini, "NLO QCD corrections to WWbb production at hadron colliders", Phys. Rev. Lett. 106 (2011) 052001, [arXiv:1012. 3975 [hep-ph]].

[28] N. Greiner, A. Guffanti, T. Reiter and J. Reuter, "NLO QCD corrections to the production of two bottom-antibottom pairs at the LHC", Phys. Rev. Lett. 107 (2011) 102002, [arXiv:1105.3624 [hep-ph]].

[29] T. Melia, K. Melnikov, R. Rontsch and G. Zanderighi, "NLO QCD corrections for $W^{+} W^{-}$ pair production in association with two jets at hadron colliders", Phys. Rev. D 83 (2011) 114043, [arXiv:1104.2327 [hep-ph]].

[30] N. Greiner, G. Heinrich, P. Mastrolia, G. Ossola, T. Reiter and F. Tramontano, "NLO QCD corrections to the production of $W^{+} W^{-}$plus two jets at the $L H C$ ", arXiv: 1202.6004 [hep-ph].

[31] B. Jager, C. Oleari and D. Zeppenfeld, "Next-to-leading order QCD corrections to $W^{+} W^{+} j j$ and $W^{-} W^{-} j j$ production via weak-boson fusion", Phys. Rev. D 80 (2009) 034022, [arXiv:0907.0580 [hep-ph]].

[32] T. Melia, K. Melnikov, R. Rontsch and G. Zanderighi, "Next-to-leading order QCD predictions for $W^{+} W^{+} j j$ production at the LHC", JHEP 1012 (2010) 053, [arXiv:1007.5313 [hep-ph]].

[33] C. F. Berger, Z. Bern, L. J. Dixon, F. Febres Cordero, D. Forde, T. Gleisberg, H. Ita and D. A. Kosower et al., "Precise Predictions for $W+3$ Jet Production at Hadron Colliders", Phys. Rev. Lett. 102 (2009) 222001, [arXiv:0902.2760 [hep-ph]].

[34] C. F. Berger, Z. Bern, L. J. Dixon, F. Febres Cordero, D. Forde, T. Gleisberg, H. Ita and D. A. Kosower et al., "Next-to-Leading Order QCD Predictions for W+3-Jet Distributions at Hadron Colliders", Phys. Rev. D 80 (2009) 074036, [arXiv:0907.1984 [hep-ph]].

[35] C. F. Berger, Z. Bern, L. J. Dixon, F. Febres Cordero, D. Forde, T. Gleisberg, H. Ita and D. A. Kosower et al., "Next-to-Leading Order QCD Predictions for Z,gamma* + 3-Jet Distributions at the Tevatron", Phys. Rev. D 82 (2010) 074002, [arXiv:1004.1659 [hep-ph]].

[36] F. Campanario, C. Englert, M. Rauch and D. Zeppenfeld, "Precise predictions for $W \gamma \gamma+j e t$ 
production at hadron colliders", Phys. Lett. B 704 (2011) 515, [arXiv:1106.4009 [hep-ph]].

[37] Z. Bern, G. Diana, L. J. Dixon, F. Febres Cordero, S. Hoeche, D. A. Kosower, H. Ita and D. Maitre et al., "Four-Jet Production at the Large Hadron Collider at Next-to-Leading Order in QCD", arXiv:1112.3940 [hep-ph].

[38] C. F. Berger, Z. Bern, L. J. Dixon, F. Febres Cordero, D. Forde, T. Gleisberg, H. Ita and D. A. Kosower et al., "Precise Predictions for $W+4$ Jet Production at the Large Hadron Collider", Phys. Rev. Lett. 106 (2011) 092001, [arXiv:1009.2338 [hep-ph]].

[39] H. Ita, Z. Bern, L. J. Dixon, F. Febres Cordero, D. A. Kosower and D. Maitre, "Precise Predictions for Z + 4 Jets at Hadron Colliders", Phys. Rev. D 85 (2012) 031501, [arXiv:1108.2229 [hep-ph]].

[40] P. Draggiotis, R. H. P. Kleiss and C. G. Papadopoulos, "On the computation of multigluon amplitudes", Phys. Lett. B 439 (1998) 157, [hep-ph/9807207].

[41] P. D. Draggiotis, R. H. P. Kleiss and C. G. Papadopoulos, "Multijet production in hadron collisions", Eur. Phys. J. C 24 (2002) 447, [hep-ph/0202201].

[42] C. G. Papadopoulos and M. Worek, "Multi-parton cross sections at hadron colliders", Eur. Phys. J. C 50 (2007) 843, [hep-ph/0512150].

[43] M. Czakon, C. G. Papadopoulos and M. Worek, "Polarizing the Dipoles", JHEP 0908 (2009) 085, [arXiv:0905.0883 [hep-ph]].

[44] A. Kanaki and C. G. Papadopoulos, "HELAC: A Package to compute electroweak helicity amplitudes", Comput. Phys. Commun. 132 (2000) 306, [hep-ph/0002082].

[45] C. G. Papadopoulos, "PHEGAS: A Phase space generator for automatic cross-section computation", Comput. Phys. Commun. 137 (2001) 247, [hep-ph/0007335].

[46] A. Cafarella, C. G. Papadopoulos and M. Worek, "Helac-Phegas: A Generator for all parton level processes", Comput. Phys. Commun. 180 (2009) 1941, [arXiv:0710.2427 [hep-ph]].

[47] A. van Hameren, "PARNI for importance sampling and density estimation", Acta Phys. Polon. B 40 (2009) 259, [arXiv:0710.2448 [hep-ph]].

[48] A. van Hameren, "Kaleu: A General-Purpose Parton-Level Phase Space Generator", arXiv:1003.4953 [hep-ph].

[49] P. Nogueira, “Automatic Feynman graph generation", J. Comput. Phys. 105 (1993) 279.

[50] A. van Hameren, C. G. Papadopoulos and R. Pittau, "Automated one-loop calculations: A Proof of concept", JHEP 0909 (2009) 106, [arXiv:0903.4665 [hep-ph]].

[51] G. Ossola, C. G. Papadopoulos and R. Pittau, "CutTools: A Program implementing the OPP reduction method to compute one-loop amplitudes", JHEP 0803 (2008) 042, [arXiv:0711.3596 [hep-ph]].

[52] P. Draggiotis, M. V. Garzelli, C. G. Papadopoulos and R. Pittau, "Feynman Rules for the Rational Part of the QCD 1-loop amplitudes", JHEP 0904 (2009) 072, [arXiv:0903.0356 [hep-ph]].

[53] G. Ossola, C. G. Papadopoulos and R. Pittau, "On the Rational Terms of the one-loop amplitudes", JHEP 0805 (2008) 004, [arXiv:0802.1876 [hep-ph]].

[54] P. Mastrolia, G. Ossola, C. G. Papadopoulos and R. Pittau, "Optimizing the Reduction of One-Loop Amplitudes", JHEP 0806 (2008) 030, [arXiv:0803.3964 [hep-ph]]. 
[55] A. van Hameren, "OneLOop: For the evaluation of one-loop scalar functions", Comput. Phys. Commun. 182 (2011) 2427, [arXiv:1007.4716 [hep-ph]].

[56] S. Catani and M. H. Seymour, "A General algorithm for calculating jet cross-sections in NLO QCD”, Nucl. Phys. B 485 (1997) 291, [Erratum-ibid. B 510 (1998) 503], [hep-ph/9605323].

[57] S. Catani, S. Dittmaier, M. H. Seymour and Z. Trocsanyi, "The Dipole formalism for next-to-leading order QCD calculations with massive partons”, Nucl. Phys. B 627 (2002) 189, [hep-ph/0201036].

[58] Z. Nagy and Z. Trocsanyi, "Next-to-leading order calculation of four jet observables in electron positron annihilation", Phys. Rev. D 59 (1999) 014020, [Erratum-ibid. D 62 (2000) 099902], [hep-ph/9806317].

[59] G. Bevilacqua, M. Czakon, M. V. Garzelli, A. van Hameren, A. Kardos, C. G. Papadopoulos, R. Pittau and M. Worek, "Helac-NLO", arXiv:1110.1499 [hep-ph].

[60] A. D. Martin, W. J. Stirling, R. S. Thorne and G. Watt, "Parton distributions for the LHC", Eur. Phys. J. C 63 (2009) 189, [arXiv:0901.0002 [hep-ph]].

[61] P. M. Nadolsky and Z. Sullivan, "PDF uncertainties in WH production at Tevatron", eConf C 010630 (2001) P510, [hep-ph/0110378].

[62] M. Cacciari, S. Frixione, M. L. Mangano, P. Nason and G. Ridolfi, "Updated predictions for the total production cross sections of top and of heavier quark pairs at the Tevatron and at the LHC", JHEP 0809 (2008) 127, [arXiv:0804.2800 [hep-ph] ].

[63] H. -L. Lai, J. Huston, S. Mrenna, P. Nadolsky, D. Stump, W. -K. Tung and C. -P. Yuan, "Parton Distributions for Event Generators", JHEP 1004 (2010) 035, [arXiv:0910.4183 [hep-ph]].

[64] H. -L. Lai, M. Guzzi, J. Huston, Z. Li, P. M. Nadolsky, J. Pumplin and C. -P. Yuan, "New parton distributions for collider physics", Phys. Rev. D 82 (2010) 074024, [arXiv:1007.2241 [hep-ph]].

[65] J. Pumplin, D. R. Stump, J. Huston, H. L. Lai, P. M. Nadolsky and W. K. Tung, “New generation of parton distributions with uncertainties from global QCD analysis, JHEP $\mathbf{0 2 0 7}$ (2002) 012, [hep-ph/0201195].

[66] D. Stump, J. Huston, J. Pumplin, W. -K. Tung, H. L. Lai, S. Kuhlmann and J. F. Owens, "Inclusive jet production, parton distributions, and the search for new physics", JHEP 0310 (2003) 046, [hep-ph/0303013].

[67] J. Alwall, S. Hoche, F. Krauss, N. Lavesson, L. Lonnblad, F. Maltoni, M. L. Mangano and M. Moretti et al., "Comparative study of various algorithms for the merging of parton showers and matrix elements in hadronic collisions", Eur. Phys. J. C 53 (2008) 473,

[arXiv:0706.2569 [hep-ph]]. 